PROCEEDINGS OF THE

AMERICAN MATHEMATICAL SOCIETY

Volume 138, Number 7, July 2010, Pages 2619-2628

S 0002-9939(10)10340-2

Article electronically published on March 15, 2010

\title{
APPROXIMATION OF PROBABILITY DISTRIBUTIONS BY CONVEX MIXTURES OF GAUSSIAN MEASURES
}

\author{
ATHANASSIA G. BACHAROGLOU \\ (Communicated by Nigel J. Kalton)
}

\begin{abstract}
Let $\mathcal{A}_{+}=\left\{a=\left(a_{n}\right) \in \bigcap_{p>1} \ell_{p}: a_{n}>0, \forall n \in \mathbb{N}\right\}$ and let $\left\{\phi_{j}\right\}_{j=1}^{\infty}$ be an enumeration of all normal distributions with mean a rational number and variance $\frac{1}{n^{2}}, n=1,2 \ldots$ We prove that there exists an $a \in \mathcal{A}_{+}$ such that every probability density function, continuous, with compact support in $\mathbb{R}$, can be approximated in $L^{1}$ and $L^{\infty}$ norm simultaneously by the averages $\frac{1}{\sum_{j=1}^{n} a_{j}} \sum_{j=1}^{n} a_{j} \phi_{j}$. The set of such sequences is a dense $G_{\delta}$ set in $\mathcal{A}_{+}$and contains a dense positive cone.
\end{abstract}

\section{INTRODUCTION}

The present work is motivated by the paper 6 of V. Nestoridis and V. Stefanopoulos, where, in the framework of universal series, they deal with the question of approximation of any random variable by a linear combination of normal distributions. More precisely, let us set $\mathcal{A}=\bigcap_{p>1} \ell_{p}$ and for $t>0$ consider the normal distribution $\phi_{t}$ on $\mathbb{R}^{d}$ :

$$
\phi_{t}(x)=\frac{e^{-\|x\|^{2} / 2 t^{2}}}{\left(2 \pi t^{2}\right)^{d / 2}}, \quad x \in \mathbb{R}^{d} .
$$

Let us denote by $\left\{\phi_{n}\right\}_{n \geq 1}$ an enumeration of all $\phi_{1 / k}\left(x-\xi_{\ell}\right), k \geq 1, \xi_{\ell} \in \mathbb{Q}^{d}$. It is proved in [6] that there exists a sequence $\alpha=\left(\alpha_{n}\right)_{n \geq 1}$ in $\mathcal{A}$ such that for every $f \in \mathcal{C}_{c}\left(\mathbb{R}^{d}\right)$, there exists an increasing sequence $\left(\lambda_{n}\right)_{n \geq 1}$ in $\mathbb{N}$ such that the partial sums

$$
\mathcal{S}_{\lambda_{n}}=\sum_{k=1}^{\lambda_{n}} \alpha_{k} \phi_{k}
$$

satisfy

$$
\left\|f f-\mathcal{S}_{\lambda_{n}} \mid\right\|:=\left\|f-\mathcal{S}_{\lambda_{n}}\right\|_{1}+\left\|f-\mathcal{S}_{\lambda_{n}}\right\|_{\infty} \rightarrow 0, \quad \text { as } n \rightarrow \infty .
$$

Furthermore, if $\mathcal{U}(\mathcal{A})$ is the class of the sequences $\alpha \in \mathcal{A}$ that satisfy relation (1), then $\mathcal{U}(\mathcal{A})$ is a dense $G_{\delta}$ set in $\mathcal{A}$ and contains a dense vector subspace of $\mathcal{A}$ except the zero sequence. Note that the terms $\alpha_{n}$ of the sequence $\alpha \in \mathcal{A}$ above cannot

Received by the editors July 15, 2009, and, in revised form, December 11, 2009.

2010 Mathematics Subject Classification. Primary 62E17; Secondary 41A30.

Key words and phrases. Mixture, probability density function, normal distribution, universal series, algebraic genericity.

This work was funded by the State Scholarships Foundation of Greece (I K Y).

(C)2010 American Mathematical Society Reverts to public domain 28 years from publication 
be all positive. Therefore, the question which naturally arises is the following. Is there a sequence $\alpha \in \mathcal{A}_{+}=\left\{a=\left(a_{n}\right) \in \mathcal{A}: a_{n}>0, \forall n \in \mathbb{N}\right\}$ for which the convex partial sums

$$
T_{n}(\alpha)=\frac{1}{\sum_{j=1}^{n} \alpha_{j}} \sum_{j=1}^{n} \alpha_{j} \phi_{j}, \quad n \in \mathbb{N},
$$

approximate every probability density function in $L^{1}$ and $L^{\infty}$ norm simultaneously? Note that the convex partial sums are also called mixtures, and from the probabilistic and statistical viewpoint it is more important to have approximations of a probability density function by mixtures of normal distributions than by arbitrary linear combinations.

The first result in this direction is given in [1, 4], where the approximation is valid in the $L^{1}$ norm.

In the present paper we strengthen the results of [1, 4. More precisely, we deal with the approximation of a probability density function $f$ on $\mathbb{R}$ by mixtures of normal distributions in $L^{1}$ and $L^{\infty}$ norm simultaneously. Our first result is the following approximation theorem.

Theorem 1.1. There exists $\alpha=\left(a_{n}\right) \in \mathcal{A}_{+}$such that for every probability density function $f \in \mathcal{C}_{c}(\mathbb{R})$ there exists a sequence of natural numbers $\left\{\lambda_{n}\right\}_{n \geq 1}$ such that

$$
\left\|\mid f-T_{\lambda_{n}}(\alpha)\right\| \rightarrow 0, \quad \text { as } n \rightarrow \infty .
$$

As we shall see below, Theorem 1.1 automatically implies approximations in several other means; for example in the $L^{q}$ norm, $q \in(1, \infty)$ provided that $f \in L^{q}$, $f \geq 0$ and $\|f\|_{1} \leq 1$.

We note that the above approximations are valid if we replace the normal distributions by translates of any approximate identity.

Let us denote by $\mathcal{U}\left(\mathcal{A}_{+}\right)$the class of all sequences $\alpha \in \mathcal{A}_{+}$which satisfies Theorem 1.1. In accordance with the abstract theory of universal series [2, 5], we now state the following result.

Theorem 1.2. (i) The class $\mathcal{U}\left(\mathcal{A}_{+}\right)$is a dense $G_{\delta}$ set in $\mathcal{A}_{+}$.

(ii) The class $\mathcal{U}\left(\mathcal{A}_{+}\right)$contains a dense positive cone of $\mathcal{A}_{+}$.

Further, we mention that similar results may be obtained in $\mathbb{R}^{d}$.

\section{Proof of Theorem 1.1}

In this section we give the proof of Theorem [1.1 Let us first mention that $\mathcal{A}$ is equipped with the metric

$$
d(a, b)=\sum_{j=1}^{\infty} \frac{1}{2^{j}} \frac{\|a-b\|_{1+\frac{1}{j}}}{1+\|a-b\|_{1+\frac{1}{j}}},
$$

where $\|.\|_{p}$ is the norm of $\ell_{p}$. With this metric $\mathcal{A}$ becomes a Frechet space. Note that $\mathcal{A}_{+}$is a $G_{\delta}$ subset of $\mathcal{A}$ and, thus $\left(\mathcal{A}_{+}, d\right)$ is a complete metric space. Moreover, to simplify notation, we denote by $X$ the space of all probability density functions with compact support, $f \in \mathcal{C}_{c}(\mathbb{R})$. Note that $X$ is a subspace of $L^{1} \cap L^{\infty}$ and that the space $L^{1} \cap L^{\infty}$ is endowed with the norm \|\|$\cdot\|\|=\|\cdot\|_{1}+\|\cdot\|_{\infty}$.

Let us recall that if

$$
\phi_{\sigma^{2}}(x-\tau)=\frac{1}{\sigma \sqrt{2 \pi}} e^{-\frac{(x-\tau)^{2}}{2 \sigma^{2}}},
$$


then $\tau$ is the mean of $\phi$ and $\sigma^{2}$ its variance.

We denote by $\left\{\phi_{j}\right\}_{j \geq 1}$ an enumeration of normal distributions with mean a rational number and variance $\frac{1}{n^{2}}, n=1,2 \ldots$.

The basic tool for the proof of Theorem 1.1 is a modified version of the following lemma of V. Nestoridis and V. Stefanopoulos in [6].

Lemma 2.1. Let $\left\{\xi_{n}\right\}_{n \geq 1}$ be an enumeration of $\mathbb{Q}^{d}$, let $p \in(1, \infty)$ and let $K \subset \mathbb{R}^{d}$ be compact. Then, given $f \in L^{\infty} \cap C\left(\mathbb{R}^{d}\right)$ and $\delta>0$, there exists $\epsilon_{0}>0$ such that for every $\epsilon \in\left(0, \epsilon_{0}\right)$ there exist $N \in \mathbb{N}$ and scalars $c_{1}, \ldots, c_{N}$ such that the following hold:

$$
\sup _{x \in K}\left|f(x)-\sum_{k=1}^{N} c_{k} \phi_{\epsilon}\left(x-\xi_{k}\right)\right|<\epsilon \quad \text { and } \quad\left(\sum_{k=1}^{N}\left|c_{k}\right|^{p}\right)^{\frac{1}{p}}<\delta .
$$

From the lemma above, Lemma 2.2 follows easily. We shall use Lemma 2.2 in the proof of Theorem 1.1.

Lemma 2.2. For every function $f \in X$ and for every $\epsilon>0$, there exist normal distributions $\phi_{1}, \ldots, \phi_{M}$ and positive numbers $b_{1}, \ldots, b_{M}$ with $\sum_{i=1}^{M} b_{i}=1$ such that

$$
\left\|f-\sum_{i=1}^{M} b_{i} \phi_{i}\right\| \mid<\epsilon .
$$

Proof. Let us recall that if $\left\{\Phi_{t}\right\}_{t>0}$ is the above-mentioned family of normal distributions and $f \in X$, then $\Phi_{t} * f$ approximates $f$ as $t \rightarrow 0$ in $L^{1}$ and $L^{\infty}$ norm simultaneously. Furthermore, each convolution

$$
f * \Phi_{t}(x)=\int_{y \in \operatorname{supp}(f)} \Phi_{t}(x-y) f(y) d y
$$

can be approximated by a Riemann sum. Therefore, there exists a cover of the support of $f$ by $\left\{V_{1}, \ldots, V_{M}\right\}$ and points $y_{1}, \ldots, y_{M}$, with $y_{k} \in V_{k}, k=1, \ldots, M$, such that

$$
\left\|\left|f * \Phi_{t}(x)-\sum_{k=1}^{M} f\left(y_{k}\right)\right| V_{k} \mid \Phi_{t}\left(x-y_{k}\right)\right\|<\frac{\epsilon}{2} .
$$

Setting $b_{i}=f\left(y_{k}\right)\left|V_{k}\right|$ in (3) where $\left|V_{k}\right|$ is the volume of $V_{k}$, the approximation (2) holds. It remains to properly choose the points $y_{k} \in V_{k}$ such that $\sum_{i=1}^{M} b_{i}=$ $\sum_{i=1}^{M} f\left(y_{k}\right)\left|V_{k}\right|=1$. Applying the mean value theorem for integration for $f$ and the connected $V_{k}$, we get the desired property easily.

We shall also use the following (see [3]).

Remark 2.3. For every finite set $I \subset \mathbb{N}$, there exist distinct indices $j_{n}(i), n \in \mathbb{N}$, $i \in I$, such that $\phi_{j_{n}(i)} \rightarrow \phi_{i}$ as $n \rightarrow \infty$.

Next we give the proof of a slightly more general version of Theorem 1.1. More precisely,

Theorem 2.4. Let $\mu$ be an infinite subset of $\mathbb{N}$. Then there exists $\alpha \in \mathcal{A}_{+}$such that for every probability density function $f \in X$, there exists a sequence $\left\{\lambda_{n}\right\}_{n \geq 1}$ in $\mu$ such that

$$
\|\| f-T_{\lambda_{n}}(\alpha)\|\| \rightarrow 0, \quad \text { as } \quad n \rightarrow \infty
$$


Proof. Let $\left\{f_{j}\right\}_{j \geq 1}$ be an enumeration of the class of functions $f$ in $X$ which are supported in $[-n, n]$; they are piecewise linear with vertices in $(\mathbb{Q}, \mathbb{Q})$. This sequence is dense in $X$.

We apply Lemma 2.2 for the function $f_{1}$ and $\epsilon=1$. Thus, there exist $b_{1}, \ldots, b_{\lambda}>$ 0 with $\sum_{j=1}^{\lambda} b_{j}=1$, and there exist normal distributions $\phi_{1_{1}}, \ldots, \phi_{1_{\lambda}}$ such that

$$
\left\|f_{1}-\sum_{j=1}^{\lambda} b_{j} \phi_{1_{j}}\right\| \mid<\frac{\epsilon}{2} .
$$

Next, by Remark 2.3, for every $j=1, \ldots, \lambda$ we can find distinct indices $j_{1}, \ldots, j_{N_{1}}$ such that

$$
\left\|\left|b_{j} \phi_{1_{j}}-\frac{b_{j}}{N_{1}} \sum_{\nu=1}^{N_{1}} \phi_{1_{j_{\nu}}} \|\right|<\frac{\epsilon}{2} .\right.
$$

From (4), (5) it follows that

$$
\left\|f_{1}-\sum_{j=1}^{\lambda} \sum_{\nu=1}^{N_{1}} \frac{b_{j}}{N_{1}} \phi_{1_{j_{\nu}}}\right\| \mid<\epsilon=1 .
$$

Let us now choose $k_{0}$ and $N_{1}$ such that $\sum_{m=k_{0}+1}^{\infty} \frac{1}{2^{m}}<\frac{\epsilon}{2}$ and

$$
\left(\sum_{j=1}^{\lambda} \frac{\left|b_{j}\right|^{p_{j}}}{N_{1}^{p_{j}-1}}\right)^{\frac{1}{p_{j}}}<\frac{\epsilon}{2}
$$

for every $p_{j}=1+\frac{1}{j}, j=1, \ldots, k_{0}$.

Since $\mu$ is an infinite subset of $\mathbb{N}$, there exists $\lambda_{1} \in \mu$ such that $\lambda_{1}>\lambda$, where $\lambda$ appears in (6). We set

$$
\beta_{j}= \begin{cases}\frac{b_{j}}{N_{1}}, & j=1, \ldots, \lambda, \\ \delta, & j=\lambda+1, \ldots, \lambda_{1},\end{cases}
$$

where $\delta>0$ small enough and such that the above inequalities (6), (77) hold for $\beta_{j}$, $j=1, \ldots, \lambda_{1}$.

We now continue in the same way and apply Lemma 2.2 for the function $f_{2}$ and $\epsilon=\frac{1}{2}$. We repeat the same process as in the first step. In the case when the set $\left\{\phi_{1_{j}}: j=1, \ldots, \lambda_{1}\right\}$ has no empty intersection with the set $\left\{\phi_{2_{j}}: j=1, \ldots, \nu\right\}$, we use Remark 2.3. Thus, we can find a natural number $\lambda_{2} \in \mu$ and scalars $\alpha_{1}, \ldots, \alpha_{\lambda_{2}}$ such that

$$
\left\|f_{2}-\sum_{j=1}^{\lambda_{2}} \alpha_{j} \phi_{2_{j}}\right\| \mid<\epsilon=\frac{1}{2} .
$$


If $L$ is a large enough positive number, then

$$
\begin{array}{r}
\left\|f_{2}-\frac{\sum_{j=1}^{\lambda_{1}} \beta_{j} \phi_{1_{j}}+L \sum_{j=1}^{\lambda_{2}} \alpha_{j} \phi_{2_{j}}}{\sum_{j=1}^{\lambda_{1}} \beta_{j}+L \sum_{j=1}^{\lambda_{2}} \alpha_{j}}\right\| \\
\sim\left\|f_{2}-\frac{L \sum_{j=1}^{\lambda_{2}} \alpha_{j} \phi_{2_{j}}}{L \sum_{j=1}^{\lambda_{2}} \alpha_{j}}\right\| \\
=\left\|f_{2}-\frac{\sum_{j=1}^{\lambda_{2}} \alpha_{j} \phi_{2_{j}}}{\sum_{j=1}^{\lambda_{2}} \alpha_{j}}\right\| \mid<\epsilon=\frac{1}{2} .
\end{array}
$$

We proceed with the infinite induction as above, applying Lemma2.2 to the function $f_{k}$ and $\epsilon=\frac{1}{k}$. In this way we build up a sequence $a \in \mathcal{A}_{+}$such that for every probability density function $f \in X$, there exists a sequence $\left\{\lambda_{n}\right\}_{n \geq 1}$ in $\mu$ such that

$$
\|\| f-T_{\lambda_{n}}(\alpha) \mid \| \rightarrow 0, \quad n \rightarrow \infty .
$$

This completes the proof of Theorem 2.4

The above theorem automatically implies approximations in several other means. Particularly we have the following.

Corollary 2.5. If $\alpha=\left(\alpha_{n}\right)_{n \geq 1} \in \mathcal{A}_{+}$is the universal sequence given in Theorem 2.4, then

(1) If $f$ is a nonnegative function in $L^{q}(\mathbb{R}) \cap \mathcal{C}(\mathbb{R})$ with $q \in(1, \infty)$ and $\int f \leq 1$, then there exists a sequence $\left(\nu_{n}\right)_{n \geq 1}$ in $\mathbb{N}$ such that

$$
\left\|f-T_{\nu_{n}}(\alpha)\right\|_{q} \rightarrow 0, \quad n \rightarrow \infty .
$$

(2) If $f$ is a nonnegative function in $L^{1}(\mathbb{R}) \cap \mathcal{C}(\mathbb{R})$ and $\int f=1$, then there exists a sequence $\left(\nu_{n}\right)_{n \geq 1}$ in $\mathbb{N}$ such that

$$
\left\|f-T_{\nu_{n}}(\alpha)\right\|_{1} \rightarrow 0, \quad n \rightarrow \infty .
$$

(3) If $f$ is a nonnegative function in $L^{\infty}(\mathbb{R}) \cap \mathcal{C}(\mathbb{R})$, vanishes at infinity and $\int f \leq 1$, then there exists a sequence $\left(\nu_{n}\right)_{n \geq 1}$ in $\mathbb{N}$ such that

$$
\left\|f-T_{\nu_{n}}(\alpha)\right\|_{\infty} \rightarrow 0, \quad n \rightarrow \infty .
$$

(4) If $f$ is a nonnegative measurable function, then there exists a sequence $\left(\nu_{n}\right)_{n \geq 1}$ in $\mathbb{N}$ such that

$$
T_{\nu_{n}}(\alpha) \rightarrow f \text { almost everywhere as } n \rightarrow \infty \text {. }
$$

\section{A topological Generic Result}

Let us denote by $\mathcal{U}_{\mu}$ the class of all sequences $\alpha \in \mathcal{A}_{+}$which satisfy Theorem 2.4 In this section, we shall prove claim (i) of Theorem 1.2, which is a topological generic result for the class $\mathcal{U}_{\mu}$. Namely, we prove the following:

Proposition 3.1. The class $\mathcal{U}_{\mu}$ is $a G_{\delta}$ and dense subset of $\mathcal{A}_{+}$.

To begin with, let us note that if we modify a finite number of terms of a sequence $\alpha \in \mathcal{U}_{\mu}$, then the modified sequence remains in $\mathcal{U}_{\mu}$. 
Lemma 3.2. Let $\alpha=\left(\alpha_{n}\right)_{n \geq 1} \in \mathcal{U}_{\mu}$ and let us set

$$
\beta=\left(\beta_{1}, \beta_{2}, \ldots, \beta_{M}, \alpha_{M+1}, \alpha_{M+2}, \ldots\right) .
$$

Then $\beta \in \mathcal{U}_{\mu}$, and if $f \in X$, there exists a sequence $\left\{\lambda_{n}\right\}_{n \geq 1}$ in $\mu$ such that

$$
\left\|\left|f-T_{\lambda_{n}}(\alpha) \|\right| \rightarrow 0, \quad n \rightarrow \infty,\right.
$$

and

$$
\left\||| f-T_{\lambda_{n}}(\beta) \mid\right\| \rightarrow 0, \quad n \rightarrow \infty .
$$

Note that (8) and (9) are valid with the same sequence $\left\{\lambda_{n}\right\}_{n=1}^{\infty}$ in $\mu$.

Proof. We first note that if $\alpha=\left(\alpha_{1}, \alpha_{2}, \ldots\right) \in \mathcal{U}_{\mu}$, then $\sum_{k=1}^{\infty} \alpha_{k}=+\infty$. Indeed, if we assume that $\sum_{k=1}^{\infty} \alpha_{k}=\Lambda<\infty$, then for a fixed point $x \in \mathbb{R}$ and for functions $f, g \in X$ such that $f(x) \neq g(x)$, there exist sequences $\left\{\lambda_{n}\right\}_{n \geq 1},\left\{\nu_{n}\right\}_{n \geq 1}$ in $\mu$ such that

$$
\lim _{n \rightarrow \infty} \sum_{j=\lambda_{1}}^{\lambda_{n}} \alpha_{j} \phi_{j}(x)=\Lambda f(x) \neq \Lambda g(x)=\lim _{n \rightarrow \infty} \sum_{j=\nu_{1}}^{\nu_{n}} \alpha_{j} \phi_{j}(x) .
$$

The above relationship is not true, since the sequence $\left\{\sum_{j=1}^{N} \alpha_{j} \phi_{j}(x)\right\}$ is an increasing sequence of positive terms and will converge to a positive number or to $\infty$.

We now give the steps of the proof. Here the role of the above observation becomes apparent, since it is crucial for the proof of Lemma 3.2 .

$$
\begin{aligned}
& \text { - }\left\|T_{\lambda_{n}}(\alpha)-\frac{1}{\sum_{k=\lambda_{1}}^{\lambda_{n}} \alpha_{k}} \sum_{k=M+1}^{\lambda_{n}} \alpha_{k} \phi_{k}\right\| \stackrel{n \rightarrow \infty}{\longrightarrow} 0 . \\
& \text { - }\left\|\frac{1}{\sum_{k=\lambda_{1}}^{\lambda_{n}} \alpha_{k}} \sum_{k=M+1}^{\lambda_{n}} \alpha_{k} \phi_{k}-\frac{1}{\sum_{k=M+1}^{\lambda_{n} n} \alpha_{k}} \sum_{k=M+1}^{\lambda_{n}} \alpha_{k} \phi_{k}\right\| \stackrel{n \rightarrow \infty}{\longrightarrow} 0 . \\
& \text { - }\left\|\frac{1}{\sum_{k=M+1}^{\lambda_{n}} \alpha_{k}} \sum_{k=M+1}^{\lambda_{n}} \alpha_{k} \phi_{k}-\frac{1}{\left(\sum_{k=1}^{M} \beta_{k}+\sum_{k=M+1}^{\lambda_{n}} \alpha_{k}\right)} \sum_{k=M+1}^{\lambda_{n}} \alpha_{k} \phi_{k}\right\| \| \\
& \quad \stackrel{n \rightarrow \infty}{\longrightarrow} 0 . \\
& \text { - }\left\|\frac{\mid}{\left(\sum_{k=1}^{M} \beta_{k}+\sum_{k=M+1}^{\lambda_{n}} \alpha_{k}\right)} \sum_{k=M+1}^{\lambda_{n}} \alpha_{k} \phi_{k}-T_{\lambda_{n}}(\beta)\right\| \stackrel{n \rightarrow \infty}{\longrightarrow} 0 .
\end{aligned}
$$

The following lemma deals with the density of the class $\mathcal{U}_{\mu}$.

Lemma 3.3. The class $\mathcal{U}_{\mu}$ is dense in $\mathcal{A}_{+}$.

Proof. We shall prove that for every $\alpha \in \mathcal{A}_{+}$and $\epsilon>0$ there exists $\gamma \in \mathcal{U}_{\mu}$ such that

$$
d(\alpha, \gamma)=\sum_{j=1}^{\infty} \frac{1}{2^{j}} \frac{\|\alpha-\gamma\|_{1+\frac{1}{j}}}{1+\|\alpha-\gamma\|_{1+\frac{1}{j}}}<\epsilon .
$$


Let $\alpha \in \mathcal{A}_{+}$and $\beta \in \mathcal{U}_{\mu}$ and let us choose $M \in \mathbb{N}$ such that

$$
\left(\sum_{j=M}^{\infty}\left|\alpha_{j}\right|^{p_{j}}\right)^{\frac{1}{p_{j}}}<\frac{\epsilon}{3} \text { and }\left(\sum_{j=M}^{\infty}\left|\beta_{j}\right|^{p_{j}}\right)^{\frac{1}{p_{j}}}<\frac{\epsilon}{3}
$$

for all $p_{j}=1+\frac{1}{j}, j=1, \ldots, M$.

Let us set

$$
\gamma=\left(\alpha_{1}, \alpha_{2}, \ldots, \alpha_{M}, \beta_{M+1}, \beta_{M+2}, \ldots\right)
$$

as a modification of $\beta$. Then by Lemma 3.2, $\gamma \in \mathcal{U}_{\mu}$ and

$$
\begin{gathered}
\|\alpha-\gamma\|_{p_{j}}=\left(\sum_{j=1}^{\infty}\left|\alpha_{j}-\gamma_{j}\right|^{p_{j}}\right)^{\frac{1}{p_{j}}}=\left(\sum_{j=M}^{\infty}\left|\alpha_{j}-\beta_{j}\right|^{p_{j}}\right)^{\frac{1}{p_{j}}} \\
\leq\left(\sum_{j=M}^{\infty}\left|\alpha_{j}\right|^{p_{j}}\right)^{\frac{1}{p_{j}}}+\left(\sum_{j=M}^{\infty}\left|\beta_{j}\right|^{p_{j}}\right)^{\frac{1}{p_{j}}}<\frac{2 \epsilon}{3}
\end{gathered}
$$

for all $p_{j}=1+\frac{1}{j}, j=1, \ldots, M$.

Let $n_{0} \in \mathbb{N}$ be such that

$$
\sum_{j=n_{0}}^{\infty} \frac{1}{2^{j}}<\frac{\epsilon}{3}
$$

and $L=\max \left\{n_{0}, M\right\}$.

From (10) and (11) we get that

$$
d(\alpha, \gamma)=\sum_{j=1}^{\infty} \frac{1}{2^{j}} \frac{\|\alpha-\gamma\|_{1+\frac{1}{j}}}{1+\|\alpha-\gamma\|_{1+\frac{1}{j}}} \leq \sum_{j=1}^{M} \frac{1}{2^{j}} \frac{\|\alpha-\gamma\|_{1+\frac{1}{j}}}{1+\|\alpha-\gamma\|_{1+\frac{1}{j}}}+\sum_{j=M+1}^{\infty} \frac{1}{2^{j}} \leq \epsilon .
$$

It remains to prove that the class $\mathcal{U}_{\mu}$ is a $G_{\delta}$ subset of $\mathcal{A}_{+}$. Let $\left\{f_{j}\right\}_{j \geq 1}$ be the sequence we used in the proof of Theorem 2.4, i.e. an enumeration of class of functions $f$ in $X$ which are supported in $[-n, n]$ and are piecewise linear with the vertices in $(\mathbb{Q}, \mathbb{Q})$. We set

$$
E(j, s, n)=\left\{\alpha=\left(\alpha_{k}\right) \in \mathcal{A}_{+}: \quad\left\|f_{j}-T_{n}(\alpha)\right\| \mid<\frac{1}{s}\right\} .
$$

The following holds.

\section{Lemma 3.4.}

$$
\mathcal{U}_{\mu}=\bigcap_{j=1}^{\infty} \bigcap_{s=1}^{\infty} \bigcup_{n \in \mu} E(j, s, n)
$$

Proof. It is obvious that

$$
\mathcal{U}_{\mu} \subset \bigcap_{j=1}^{\infty} \bigcap_{s=1}^{\infty} \bigcup_{n \in \mu} E(j, s, n)
$$

In order to prove the reverse inclusion we consider a sequence

$$
\alpha \in \bigcap_{j=1}^{\infty} \bigcap_{s=1}^{\infty} \bigcup_{n \in \mu} E(j, s, n)
$$


and $f \in X$. Since $\left\{f_{j}\right\}_{j \geq 1}$ is a dense sequence in $X$, for each $s \in \mathbb{N}_{0}$ there exists a function $f_{j_{s}}$ such that

$$
|| f-f_{j_{s}}||<\frac{1}{2 s}
$$

Since $\alpha \in \bigcap_{j=1}^{\infty} \bigcap_{s=1}^{\infty} \bigcup_{n \in \mu} E(j, s, n)$, there exists a sequence $\left\{n_{s}\right\}$ of nonnegative integers in $\mu$ such that for each $s \in \mathbb{N}_{0}$

$$
||\left|f_{j_{s}}-T_{n_{s}}(\alpha)\right|||<\frac{1}{2 s}
$$

Then, by (12) and (13) we get that

$$
\left\|\left|f-T_{n_{s}}(\alpha)\right|\right\|<\frac{1}{s}
$$

and the lemma follows by letting $s \rightarrow \infty$.

Lemma 3.5. For every $j, s \in \mathbb{N}_{0}$ and $n \in \mu$, the set $E(j, s, n)$ is an open subset of $\mathcal{A}_{+}$.

Proof. Let $\alpha^{0}=\left(\alpha_{k}^{0}\right) \in E(j, s, n)$ and let $\left\{\alpha^{m}\right\}_{m \geq 1}$ be a sequence of elements in $\mathcal{A}_{+}$converging to $\alpha^{0}$. To prove that $E(j, s, n)$ is open it suffices to prove that there exists $m_{0} \in \mathbb{N}_{0}$ such that, for all $m \geq m_{0}, \alpha^{m} \in E(j, s, n)$.

Since $\alpha^{m} \rightarrow \alpha^{0}$, it follows easily that

$$
\lim _{m \rightarrow \infty}\left\||| T_{n}\left(\alpha^{0}\right)-T_{n}\left(\alpha^{m}\right)\right\| \|=0 .
$$

For

$$
\left.\epsilon=\frac{1}{s}-\| \mid T_{n}\left(\alpha^{0}\right)-f_{j}\right)\|\|
$$

there exists $m_{0} \in \mathbb{N}_{0}$ such that, for all $m \geq m_{0}$, we have

$$
\left\|\left|T_{n}\left(\alpha^{0}\right)-T_{n}\left(\alpha^{m}\right) \|\right|<\epsilon\right.
$$

Then for every $m \geq m_{0}$ we have

$$
\left\|\left|T_{n}\left(\alpha^{m}\right)-f_{j}\left\|\left|\leq\left\|\left|T_{n}\left(\alpha^{0}\right)-T_{n}\left(\alpha^{m}\right)\right|\right\|\right|+|| \mid T_{n}\left(\alpha^{0}\right)-f_{j}\right)\right|||<\frac{1}{s},\right.
$$

which implies that $\alpha^{m} \in E(j, s, n)$, and the proof is complete.

Proof of Proposition 3.1. The proof follows from Lemmas 3.3, 3.4 and 3.5.

Remark 3.6. If $\phi_{j}$ are more generally the $q$-translations $(q \in \mathbb{Q})$ of an arbitrary approximate identity, then the analogue of Proposition 3.1 is valid. To obtain this stronger result one can avoid the constructive proof of Theorem 1.1 and directly use Baire's category theorem to obtain topological genericity. However the approach we have followed is more direct.

\section{An Algebraic Generic Result}

In this section we prove claim (ii) of Theorem 1.2, which implies algebraic genericity of the class $\mathcal{U}_{\mu}$. More precisely we will prove that the class $\mathcal{U}_{\mu}$ contains a dense positive cone of $\mathcal{A}_{+}$.

Let $K=\left\{\alpha^{m}\right\}_{m \geq 1}$ be a subset of $\mathcal{A}_{+}$. We first recall that the positive cone $\mathcal{C}(K)$ generated by $K$ is defined as the smallest positive cone that contains $K$ and is equal to the set

$$
\left\{c_{1} \alpha^{1}+c_{2} \alpha^{2}+\cdots+c_{m} \alpha^{m}: \quad c_{j}>0, \quad \alpha^{j} \in K, \quad 1 \leq j \leq m, \quad m \in \mathbb{N}_{0}\right\} .
$$


We first give the following lemma. Its proof is straightforward and thus omitted.

Lemma 4.1. For $\alpha^{j} \in \mathcal{A}_{+}$and $c_{j}>0,1 \leq j \leq m$, we set $\alpha=\sum_{j=1}^{m} c_{j} \alpha^{j}$ and $\beta_{N}=\sum_{j=1}^{N}\left(c_{1} \alpha_{j}^{1}+\cdots+c_{m} \alpha_{j}^{m}\right)$. Then

$$
T_{N}(\alpha)=T_{N}\left(\alpha^{1}\right) \frac{\sum_{j=1}^{N} c_{1} \alpha_{j}^{1}}{\beta_{N}}+T_{N}\left(\alpha^{2}\right) \frac{\sum_{j=1}^{N} c_{2} \alpha_{j}^{2}}{\beta_{N}}+\cdots+T_{N}\left(\alpha^{m}\right) \frac{\sum_{j=1}^{N} c_{m} \alpha_{j}^{m}}{\beta_{N}}
$$

and

$$
\sum_{i=1}^{m} \sum_{j=1}^{N} \frac{c_{i} \alpha_{j}^{i}}{\beta_{N}}=1 .
$$

Proposition 4.2. The class $\mathcal{U}_{\mu}$ contains a dense positive cone of $\mathcal{A}_{+}$.

Proof. The proof follows the steps of the proof of Theorem 3 in [2, p. 427], and it will only be sketched.

Let us fix a dense sequence $\left\{\gamma^{i}\right\}_{i \geq 1}$ in $\mathcal{A}_{+}$and let $\left\{f_{j}\right\}_{j \geq 1}$ be the dense sequence of functions in $X$ we used in the proof of Theorem [2.4. Arguing as in 2, we construct inductively a sequence $\left\{\alpha^{\nu}\right\}_{\nu \geq 1} \subset \mathcal{A}_{+}$and sequences $\mu_{j}^{\nu}, j, \nu \geq 1$, of subsets of $\mu$ such that for every $j, \nu \geq 1$ the following conditions hold true:

(i) $\mu_{j}^{\nu}$ is a subsequence of $\mu_{j}^{\nu-1}$,

(ii) $d\left(\alpha^{\nu}, \gamma^{\nu}\right)<\frac{1}{\nu}$,

(iii) $\alpha^{\nu} \in \bigcap_{j \in \mathbb{N}_{0}} \mathcal{U}_{\mu_{j}^{\nu-1}}$ and

(iv) $\lim _{n \rightarrow \infty}\left\||| f_{j}-T_{\mu_{j}^{\nu}}\left(\alpha_{n}^{\nu}\right) \mid\right\|=0$.

Denote by $C$ the positive cone in $\mathcal{A}_{+}$generated by $\left\{\alpha^{\nu}\right\}_{\nu \geq 1}$. We shall prove that $C \subset \mathcal{U}_{\mu}$. For this it suffices to show that for any $\alpha \in C$ and for any $f_{j}$ there exists a sequence $\left\{\lambda_{n}\right\} \subset \mu$ such that

$$
\lim _{n \rightarrow \infty} \mid\left\|f_{j}-T_{\lambda_{n}}(\alpha)\right\| \|=0 .
$$

But $\alpha=\sum_{i=1}^{m} c_{i} \alpha^{i}$ with $c_{i}>0$ and $\alpha^{i} \in \mathcal{U}_{\mu_{j}^{i-1}}$. Combining the fact that $\alpha^{m} \in \mathcal{U}_{\mu_{j}^{m-1}}$ with the property (i) of the family $\mu_{j}^{\nu}$, we find that there exists a sequence $\left\{\lambda_{n}\right\} \subset \mu_{j}^{m-1} \subset \mu_{j}^{m-2} \subset \cdots \subset \mu_{j}^{1} \subset \mu$ such that for every $k=1,2, \ldots, m$

$$
\lim _{n \rightarrow \infty}\left\||| f_{j}-T_{\lambda_{n}}\left(\alpha^{k}\right)\right\| \|=0 .
$$

Using (14) and Lemma 4.1 we get that $\alpha \in \mathcal{U}_{\mu}$, and the proof of the proposition is complete.

\section{ACKNOWLEDGMENTS}

The author would like to express her gratitude to V. Nestoridis for suggesting the problem and other valuable suggestions and to M. Marias for his encouragement and help in improving the presentation of this paper.

\section{REFERENCES}

[1] D. L. Alspach and H. W. Sorenson, Nonlinear Bayesian estimation using Gaussian sum approximations. IEEE Trans. Automatic Control AC-17, no. 4 (1972), 439-448.

[2] F. Bayart, K.-G. Grosse-Erdmann, V. Nestoridis and C. Papadimitropoulos, Abstract theory of universal series and applications. Proc. London Math. Soc. 96, no. 2 (2008), 417-463. MR2396846 (2009j:30006) 
[3] S. Koumandos, V. Nestoridis, Y-S. Smyrlis and V. Stefanopoulos, Universal series in $\bigcap_{p>1} \ell^{p}$, Bull. London Math. Soc. 42 (2010), 119-129.

[4] J. T. H. Lo, Finite dimensional sensor orbits and optimal nonlinear filtering. IEEE Trans. Information Theory IT-18, no. 5 (1972), 583-589. MR0386822 (52:7671)

[5] V. Nestoridis and C. Papadimitropoulos, Abstract theory of universal series and an application to Dirichlet series. C. R. Acad. Sci Paris 341, no. 9 (2005), 539-543. MR 2181390 (2006h:11108)

[6] V. Nestoridis and V. Stefanopoulos, Universal series and approximate identities, submitted (2008).

Department of Mathematics, Aristotle University of Thessaloniki, Thessaloniki 541 24, GREECE

E-mail address: ampachar@math.auth.gr 